\title{
A VISITA TÉCNICA SOB O OLHAR DOS DISCENTES DO CURSO SUPERIOR DE TECNOLOGIA EM ANÁLISE E DESENVOLVIMENTO DE SISTEMAS
}

\author{
Herika De Souza Coutinho1 \\ ORCID: 0000-0002-6404-8228 \\ Gabriela Gonçalves Barbosa2 \\ ORCID:0000-0002-2756-5461 \\ Diego Dos Santos Rodrigues 3 \\ ORCID: 0000-0001-6898-6142 \\ HÉLIO TOSHIO KAMAKAWA4
ORCID: 0000-0001-9246-0060
}

Resumo: Por meio do projeto de extensão, os acadêmicos do Curso Superior de Tecnologia em Análise e Desenvolvimento de Sistemas do Instituto Federal do Paraná (IFPR) de Paranavaí, realizaram visitas técnicas (VT) em 2 empresas de desenvolvimento de software. Objetivou-se oferecer aos acadêmicos, a possibilidade de observar a dinâmica dos profissionais do setor e entender os quesitos necessários para atuar nesta profissão. Trata-se de um estudo descritivo com abordagem qualitativa, avaliando se as VT atenderam os objetivos das atividades de extensão do IFPR na percepção dos acadêmicos. Os resultados indicam que a VT é uma ação de extensão de suma importância ao ensino, que permitiu aos acadêmicos visualizar a aplicação dos conceitos aprendidos de forma interdisciplinar no contexto real da profissão,

1 Acadêmica do Curso Superior de Tecnologia em Análise e Desenvolvimento de Sistemas do IFPR de Paranavaí

2 Acadêmica do Curso Superior de Tecnologia em Análise e Desenvolvimento de Sistemas do IFPR de Paranavaí

3 Acadêmico do Curso Superior de Tecnologia em Análise e Desenvolvimento de Sistemas do IFPR de Paranavaí

4 Professor EBTT IFPR de Paranavaí E-mail: helio.kamakawa@ifpr.edu.br 
ampliando o universo de referência que ensejam e ao mesmo tempo estreitando as relações entre a instituição com as empresas para atuar em conjunto em busca de atender as demandas da sociedade.

Palavras-chave: Visita técnica. Desenvolvimento de software. Extensão.

\title{
THE INFLUENCE OF THE TECHNICAL VISIT TO THE ACADEMIC MOTIVATION OF THE TECHNOLOGY COURSE IN SYSTEM ANALYSIS AND DEVELOPMENT
}

\begin{abstract}
Through the extension project, the academics of the Higher Course of Technology in Analysis and System Development of the Federal Institute of Paraná (IFPR) of Paranavaí, made technical visits (VT) in 2 software development companies. The aim was to offer academics the possibility of observing the dynamics of professionals in the sector and understand the requirements needed to work in this profession. This is a descriptive study, with a qualitative approach, assessing if the VT met the objectives of the extension activities of the IFPR in the perception of the academics. The results indicate that the VT is an extension action of paramount importance to teaching that allowed the students to visualize the application of the concepts learned, in an interdisciplinary way, in the real context of the profession, expanding the universe of reference that they provide and at the same time, narrowing the relations between the institution and the companies, to work together to meet the demands of society. Keywords: Technical visit. Software development. Extension.
\end{abstract}

\section{WHATSAPP ${ }^{\circledR}$ COMO HERRAMIENTA DE PROMOCIÓN DE LA SALUD EN EL DIABETES: RELATO DE EXPERIENCIA}

Resumen: Por medio del proyecto de extensión, los académicos del Curso Superior de Tecnología en Análisis y Desarrollo de Sistemas del Instituto Federal de Paraná (IFPR) de Paranavaí, realizaron visitas técnicas (VT) en 2 empresas de desarrollo de software. Se objetivó ofrecer a los académicos, la posibilidad de observar la dinámica de los profesionales del sector y entender los requisitos necesarios para actuar en esta profesión. Se trata de un estudio descriptivo, con abordaje cualitativo, evaluando si las VT atendieron los objetivos de las actividades de extensión del IFPR en la percepción de los académicos. Los resultados indican que la VT es una acción de extensión de suma importancia a la enseñanza que permitió a los académicos, visualizar la aplicación de los conceptos aprendidos, de forma interdisciplinaria, en el contexto real de la profesión, ampliando el universo de referencia que al mismo tiempo, estrechando las relaciones entre la institución con las empresas, para actuar en conjunto en busca de atender las demandas de la sociedad.

Palabras clave: Visita técnica. Desarrollo de software. Extensión. 


\section{INTRODUÇÃO}

O Instituto Federal do Paraná (IFPR) de Paranavaí oferta o Curso Superior de Tecnologia em Análise e Desenvolvimento de Sistemas desde 2013. O objetivo do curso é formar estudantes com conhecimentos técnicos para analisar, projetar e construir aplicações de software, bem como administrar e padronizar as especificações dos documentos (COMISSÃO DE ELABORAÇÃO DO PPC, 2013).

Porém, a aprendizagem de programação de software requer características que para muitos alunos são distantes do cotidiano, pois exige a escrita de instruções com regras e métodos rigorosos e a busca de soluções com uso de lógica por meio de diversas maneiras de codificar, o que gera elevados níveis de insucesso em qualquer nível e sistema de ensino (GOMES; HENRIQUES; MENDES, 2008).

Souza, Batista e Barbosa (2016), realizaram um mapeamento sistêmico a respeito de estudos relacionados às dificuldades de aprendizagem na programação, no qual, foi identificada a predominância de problemas relativos à aprendizagem de conceitos de programação, seguido de problemas relacionados à aplicação desses conceitos na construção de programas e de motivação. Os autores constataram que os artigos referentes a essas três categorias correspondem a mais de $80 \%$ do total de artigos selecionados e que foi possível observar que os problemas motivacionais estão relacionados com as dificuldades na aprendizagem e aplicação dos conceitos de programação.

Dutra et al. (2019) afirmam que, atualmente, os jovens vivenciam em seus lares, aspectos hierárquicos menos rígidos, bem como experimentaram acesso rápido e fácil à informação, e assim, não respondem a modelos educativos tradicionais centrados no professor por meio de aulas expositivas. Desta forma, segundo os autores, novos desafios são impostos aos professores no sentido de buscar alternativas capazes de resgatar o papel da educação em um contexto tecnológico e promover a construção de aprendizagem significativa e transformadora.

Uma maneira de auxiliar no processo de ensino-aprendizagem são as ações de extensão, nos quais, caracterizam-se, necessariamente, pelo envolvimento dos acadêmicos e têm por finalidade promover a interdisciplinaridade, interprofissionalidade e o impacto na formação do estudante (CONSELHO SUPERIOR DO IFPR, 2018). 
Por meio da atividade de extensão é possível compartilhar conhecimentos ao invés de transmiti-los e a partir disso, desenhar uma trajetória formativa que valorize a teoria confrontada e refletida com a prática, dando valor ao conhecimento interdisciplinar para superar uma visão fragmentada do mundo, possivelmente passada a partir da divisão das disciplinas do curso (LIMA; AZEVEDO; AMORIN, 2015).

A Visita Técnica (VT) é uma das atividades de extensão de uma instituição de ensino que pode ser realizada para que os alunos vivenciem a profissão na qual pretendem atuar em cenários reais, contribuindo na sua formação (LIMA; AZEVEDO e AMORIN, 2015; BADARÁ et al., 2016; FERKO e CAMPÊLO, 2016).

Desta forma, por meio de um dos programas de extensão do IFPR de Paranavaí, visitas técnicas foram organizadas para os alunos do Curso Superior de Tecnologia em Análise e Desenvolvimento de Sistemas (CSTADS). Objetivou-se oferecer aos acadêmicos, a possibilidade de observar a dinâmica dos profissionais do setor e entender os quesitos necessários para atuar nesta profissão. Trata-se de um estudo descritivo com abordagem qualitativa, avaliando se as VT atenderam os objetivos das atividades de extensão do IFPR na percepção dos acadêmicos.

Este estudo é constituído de oito tópicos. O primeiro apresentou o contexto da pesquisa, do objetivo e das justificativas que motivaram este estudo. O segundo tópico é dedicado à apresentação da revisão de literatura, considerando as pesquisas existentes e trabalhos correlatos nos últimos cinco anos. Os objetivos das atividades de extensão no contexto do IFPR estão descritos no terceiro tópico. No quarto tópico, estão descritas as características dos participantes da VT. A metodologia aplicada e a descrição da VT realizada estão respectivamente no quinto e sexto tópico. $\mathrm{O}$ tópico sete apresenta os resultados obtidos e o último, a discussão dos resultados e as considerações finais.

\section{REVISÃO DE LITERATURA}

Inicialmente, realizou-se a revisão de literatura para compreender o estágio atual do tema, bem como, compreender a organização e o planejamento a respeito de VT. Como fonte de pesquisa, utilizou-se o Google Acadêmico. $\mathrm{Na}$ consulta realizada em 10 de fevereiro de 2019, foi utilizado o descritor "Visita Técnica" como termo da pesquisa. Por meio da busca avançada, 
definiu-se as seguintes opções: (1) "Palavra exata" e (2) "Em qualquer lugar" indicando a busca em títulos, no autor e nos assuntos. Com estas definições, foram apresentados aproximadamente 15.500 trabalhos.

Para refinar a busca, outros critérios foram adotados, como a retirada de patentes e citações; e publicações recentes - 5 anos. Por meio destes critérios, o resultado foi aprimorado em aproximadamente 8.550 artigos. Nota-se a abundância de estudos a respeito de VT publicados em anais e eventos, dentre os quais, apresentam poucas contribuições nos procedimentos realizados, metodologia aplicada e avalição.

Em seguida, iniciou-se a seleção de artigos por meio da leitura de seus títulos. Nesta fase, como critério de inclusão, foram adicionados os estudos que abordaram de alguma forma os seguintes descritores: (1) visita técnica, (2) visitas em empresas e/ou (3) projeto de extensão. De acordo com estes critérios foram selecionados 85 artigos.

Segundo a norma NBR 6028 da Associação Brasileira de Normas Técnicas (ASSOCIAÇÃO BRASILEIRA DE NORMAS TÉCNICAS, 2003, p.1), "o resumo corresponde à apresentação concisa dos pontos relevantes de um documento". Desta forma, foi realizada a leitura pormenorizada dos resumos, descartando-se pesquisas que não correspondiam ao estudo. Dos 85 resumos analisados, verificou-se que 18 artigos faziam referência a VT.

Os estudos cujos resumos que atendiam aos critérios definidos foram lidos plenamente, no qual, 3 foram selecionados pelo fato de ser um artigo completo, estar alinhado com o escopo deste estudo, publicado em um periódico com a área de avaliação condizente com a linha de pesquisa e possuir reconhecimento de qualidade (qualis) intelectual conforme procedimentos utilizados pela CAPES. O Quadro 1 apresenta o resultado da seleção de artigos a respeito do AVA no ensino presencial.

\begin{tabular}{|c|c|c|c|}
\hline ANO & AUTORES & REVISTA & REVISTA \\
\hline 2019 & DUTRA ET AL. & $\begin{array}{l}\text { ENFERMACEM DO } \\
\text { CENTRO-OESTE } \\
\text { MINEIRO }\end{array}$ & $\begin{array}{c}\text { UTILIZAÇÃO DA VISITA TÉCNICA NO } \\
\text { ENSINO DE ADMINISTRAÇÃO EM } \\
\text { ENFERMACEM }\end{array}$ \\
\hline 2017 & FABRI E DUTRA & $\begin{array}{l}\text { ONLINE BRAZILIAN } \\
\text { JOURNAL OF } \\
\text { NURSING }\end{array}$ & $\begin{array}{l}\text { TECHNICAL VISIT CARRIED OUT DURINC } \\
\text { THE FORMATION OF NURSINC STUDENTS: A } \\
\text { DESCRIPTIVE STUDY }\end{array}$ \\
\hline 2016 & CAMPÊLO E FERKO & $\begin{array}{l}\text { ADMINISTRAÇÃO DE } \\
\text { RORAIMA }\end{array}$ & $\begin{array}{c}\text { A VISITA TÉCNICA SOB O OLHAR DOS } \\
\text { DISCENTES DO MBA EM GESTÃO DE } \\
\text { COOPERATIVA DA UFRR }\end{array}$ \\
\hline
\end{tabular}


Conforme ilustrado no Quadro 1, o primeiro estudo é de Dutra et al (2019), no qual, relata a experiência da realização de VT no ensino de administração em Enfermagem. Segundo os autores, a realização das visitas aos serviços de apoio permitiu aos estudantes desenvolverem habilidades de planejamento, tomada de decisão, trabalho em equipe e organização; bem como aprimorar o pensamento crítico-reflexivo, além de identificar a importância do trabalho intersetorial e multiprofissional para a assistência de qualidade.

O segundo estudo, escrito por Fabri e Dutra (2017), identifica se as VTs contribuem para a formação e gestão em enfermagem. Um estudo descritivo cujos dados foram obtidos em entrevistas semiestruturadas com onze estudantes do curso de graduação em enfermagem. Após transcrever as entrevistas, utilizou-se a análise de conteúdo. De acordo com os resultados, os autores concluem que a VT é uma ferramenta de ensino eficaz para promover uma abordagem à realidade do mercado de trabalho, diminuindo a distância entre teoria e prática.

O último estudo ilustrado no Quadro 1, foi realizado pelo Campêlo e Ferko (2016), no qual, apresentou a percepção dos discentes sobre a prática da visita técnica no Curso de MBA em cooperativismo. Segundo o estudo, a VT é uma atividade agregadora, pois permitiu observar o ambiente real de uma cooperativa, além de ser possível verificar sua dinâmica organizacional.

\section{OBJETIVOS DO PROJETO DE EXTENSÃO NO CONTEXTO DO IFPR}

Esta seção apresenta as normas disciplinares, que possui metas, objetivos, pressupostos teóricos e princípios que norteiam a realização das atividades de extensão no âmbito do IFPR, utilizadas como base neste estudo para a elaboração do questionário de avalição da VT.

O IFPR deve estar presente em diferentes unidades da federação, com finalidades e características de desenvolver atividades de extensão, conforme os princípios da educação profissional e tecnológica (PRESIDÊNCIA DA REPÚBLICA; CASA CIVIL; SUBCHEFIA PARA ASSUNTOS JURÍDICOS, 2008).

No âmbito do IFPR, as atividades de extensão estão regulamentadas por meio da resolução $n^{\circ} 11$, de 27 de março de 2018. A extensão é uma ação processual contínua, educativo e tecnológico, de caráter multidisciplinar, que 
promove, de forma indissociável ao ensino e à pesquisa, a interação entre o IFPR e a sociedade (CONSELHO SUPERIOR DO IFPR, 2018). Os objetivos descritos na resolução são:

i. Indissociabilidade do ensino, pesquisa e extensão - reafirmando a extensão como processo acadêmico em que toda ação de extensão está vinculada ao processo de formação das pessoas e de geração de conhecimento, tendo o acadêmico como protagonista de sua formação técnica para obtenção de competências necessárias à atuação profissional, e de sua formação cidadã.

ii. Interdisciplinaridade e interprofissionalidade - interação de modelos e conceitos complementares, de ações que busque consistência teórica confrontada com a realidade profissional, dando valor ao conhecimento interdisciplinar;

iii. Impacto na Formação do Estudante - atividades de extensão que ampliem o universo de referência que ensejam, pelo contato direto com as grandes questões contemporâneas, possibilitando o enriquecimento da experiência discente em termos teóricos e metodológicos; e

iv. Interação Dialógica - desenvolvimento de relações entre a instituição e setores públicos e/ou privados, marcadas pelo diálogo e troca de experiências, em busca de atender as demandas da sociedade.

A extensão é uma ação processual contínua, de caráter educativo, social, cultural ou tecnológico, com objetivo específico e prazo determinado, e a partir de objetivos claros e tangíveis, poderá prever diferentes atividades.

\section{PARTICIPANTES DA VISITA TÉCNICA}

A VT foi realizada com a participação de 22 alunos da $2^{\circ}$ e $3^{\circ}$ série do CSTADS, nos quais, 12 já haviam realizado uma visita técnica associado a área do curso. Da $2^{\circ}$ série, participaram 8 alunos, dentre os quais, 7 eram do sexo masculino e 1 feminino. Já na $3^{\circ}$ série, participaram 14 alunos, dentre os quais, 10 eram do sexo masculino e 4 femininos.

Dentre os 22 alunos que participaram da VT, 13 conquistaram a vaga do curso por meio de cota de inclusão social e 9 pela ampla concorrência. Vinte alunos estudaram integralmente em escolas públicas e 2 estudaram em parte, nas escolas privadas. 
Em relação as dificuldades das disciplinas técnicas, 13 alunos alegaram ter dificuldades, nos quais, 7 disseram que são oriundas pela falta de tempo, 6 pela aprendizagem dos conceitos, 3 na aplicação destes conceitos e/ou 6 pelos fatores motivacionais. Constatamos que 6 alunos possuem dependências, nos quais, 5 são em disciplinas técnicas.

Atualmente, 16 dos 22 alunos que participaram da VT trabalham, 9 correlatas nas áreas técnicas do curso. Em relação a expectativa dos alunos a respeito da VT antes de ser realizada, 17 alunos afirmaram esperar conhecer o ambiente de trabalho das empresas, a metodologia de trabalho, cargos e funções; 8 alunos disseram esperar entender quais tecnologias estão sendo utilizadas; 7 alunos disseram esperar entender quais são as competências necessárias para atuar na área; 2 para vivenciar novas experiências; e/ou 2 para buscar informações do mercado de tralho e assim, avaliar a escolha da profissão.

\section{METODOLOGIA}

Trata-se de uma pesquisa descritiva com abordagem qualitativa, avaliando se a VT realizada alcançou os objetivos definidos no regulamento das atividades de extensão do IFPR na perspectiva dos acadêmicos participantes. Como fontes de coleta de dados, foram aplicados questionários aos 22 acadêmicos do CSTADS envolvidos nas VT.

As VT foram realizadas em duas empresas do setor de Tecnologia da Informação (TI) dentro do cenário nacional, inerente ao propósito do programa de extensão e em concordância com os objetivos do CSTADS.

Após a realização das VT, a avaliação foi aplicada utilizando a ferramenta Google Forms. Na elaboração do questionário de avaliação da VT, utilizou-se como base dos critérios, os objetivos definidos na resolução $n^{\circ} 11$, de 27 de março de 2018, documento norteador, que possui metas, objetivos, pressupostos teóricos e princípios para as atividades de extensão do IFPR (CONSELHO SUPERIOR DO IFPR, 2018). O Quadro 2 apresenta o questionário de avaliação da VT. 


\begin{tabular}{|c|c|}
\hline \multicolumn{2}{|c|}{ OBJETIVO: INDISSOCIABILIDADE DO ENSINO, PESQUISA E EXTENSÃO } \\
\hline QUESTÕES ELABORADAS: & OBJETIVO dA AVALIAÇÃo \\
\hline $\begin{array}{l}\text { FOI POSSÍVEL IDENTIFICAR O QUE SE } \\
\text { APRENDE NA INSTITUIÇÃO NAS EMPRESAS } \\
\text { VISITADAS? }\end{array}$ & $\begin{array}{l}\text { AVALIAR A RELAÇÃO DO QUE É ENSINADO NA } \\
\text { INSTITUIÇÃO COM O PRATICADO NAS EMPRESAS } \\
\text { VISITADAS }\end{array}$ \\
\hline $\begin{array}{l}\text { O CURSO QUE VOCÊ ESTÁ REALIZANDO É } \\
\text { IMPORTANTE PARA ATUAR NO MERCADO DE } \\
\text { TRABALHO? }\end{array}$ & $\begin{array}{l}\text { AVALIAR NA PERCEPÇÃO DOS ACADÊMICOS, A } \\
\text { IMPORTÂNCIA DO CURSO NO CONTEXTO DAS } \\
\text { EMPRESAS VISITADAS. }\end{array}$ \\
\hline $\begin{array}{l}\text { CONHECER O AMBIENTE DE TRABALHO DA } \\
\text { PROFISSÃO, NO QUAL, SÃO ABORDADAS NAS } \\
\text { DISCIPLINAS DO CURSO AUXILIA NO SEU } \\
\text { APRENDIZADO? }\end{array}$ & $\begin{array}{l}\text { AVALIAR SE A VT AUXILIA NO APRENDIZADO } \\
\text { ACADÊMICO. }\end{array}$ \\
\hline $\begin{array}{l}\text { O QUE SE ENSINA NA INSTITUIÇÃO É } \\
\text { CONDIZENTE COM QUE FOI VISTO NAS } \\
\text { EMPRESAS VISITADAS? }\end{array}$ & $\begin{array}{l}\text { AVALIAR SE O ENSINO TÉCNICO É CONVERGENTE A } \\
\text { REALIDADE PROFISSIONAL. }\end{array}$ \\
\hline \multicolumn{2}{|c|}{ OBJETIVO: INTERDISCIPLINARIDADE E INTERPROFISSIONALIDADE. } \\
\hline QUESTÕES ELABORADAS: & OBJETIVO dA AVALIAÇÃo \\
\hline $\begin{array}{l}\text { AS DISCIPLINAS DO CURSO ABORDAM } \\
\text { CONTEÚDOS NECESSÁRIOS PARA ATUAR EM } \\
\text { UMA EMPRESA DE SOFTWARE? }\end{array}$ & $\begin{array}{l}\text { AVALIAR A RELEVÂNCIA DAS DISCIPLINAS NO } \\
\text { AMBIENTE PROFISSIONAL. }\end{array}$ \\
\hline $\begin{array}{l}\text { HÁ INTECRAÇÃO ENTRE OS CONTEÚDOS } \\
\text { VISTOS NAS DISCIPLINAS DO CURSO NO } \\
\text { AMBIENTE DE TRABALHO VISTO? }\end{array}$ & $\begin{array}{l}\text { IDENTIFICAR A INTERDISCIPLINARIDADE NO } \\
\text { AMBIENTE DE TRABALHO. }\end{array}$ \\
\hline $\begin{array}{l}\text { UM PROFISSIONAL POSSUI COMPETÊNCIAS } \\
\text { E/OU CONHECIMENTOS DE DIVERSAS } \\
\text { ÁREAS? }\end{array}$ & $\begin{array}{l}\text { AVALIAR SE A VT PROPORCIONOU A VISÃO } \\
\text { INTERPROFISSIONAL. }\end{array}$ \\
\hline \multicolumn{2}{|c|}{ OB]ETIVO: IMPACTO NA FORMAÇÃO DO ESTUDANTE } \\
\hline QUESTÕES ELABORADAS: & OBJETIVO dA AVALIAÇÃo \\
\hline $\begin{array}{l}\text { A SUA PERSPECTIVA (AMBIENTE, } \\
\text { METODOLOCIA, CARGOS, FUNÇÕES, POSTOS } \\
\text { DE TRABALHO) EM RELAÇÃO A PROFISSÃO } \\
\text { MUDOU? }\end{array}$ & $\begin{array}{l}\text { AVALIAR SE A VT CONTRIBUI NA PERCEPÇÃO DA } \\
\text { REALIDADE PROFISSIONAL. }\end{array}$ \\
\hline A VT CONTRIBUIU NA SUA FORMAÇÃO? & $\begin{array}{l}\text { AVALIAR SE VT CONTRIBUIU NA FORMAÇÃO DO } \\
\text { ACADÊMICO. }\end{array}$ \\
\hline $\begin{array}{l}\text { COM A VT, VOCÊ MUDOU NA ESCOLHA DA } \\
\text { PROFISSÃO? }\end{array}$ & $\begin{array}{l}\text { AVALIAR SE A VT IMPACTOU NA ESCOLHA DA } \\
\text { PROFISSÃO. }\end{array}$ \\
\hline $\begin{array}{l}\text { A SUA MOTIVAÇÃO NOS ESTUDOS } \\
\text { MELHOROU APÓS A VT? }\end{array}$ & $\begin{array}{l}\text { AVALIAR SE A VT CONTRIBUIU NA MOTIVAÇÃO AOS } \\
\text { ESTUDOS. }\end{array}$ \\
\hline $\begin{array}{l}\text { PARA A SUA FORMAÇÃO ACADÊMICA, A VT É } \\
\text { IMPORTANTE? }\end{array}$ & COMPREENDER AS CONTRIBUIÇÕES DA VT. \\
\hline \multicolumn{2}{|l|}{ OBJETIVO: INTERAÇÃO DIALÓGICA } \\
\hline QUESTÕES ELABORADAS: & OBJETIVO dA AVALIAÇÃo \\
\hline $\begin{array}{l}\text { A VT PROMOVEU A INTERAÇÃO DIALÓCICA } \\
\text { ENTRE A INSTITUIÇÃO COM AS EMPRESAS } \\
\text { VISITADAS? }\end{array}$ & $\begin{array}{l}\text { AVALIAR SE A VT PROMOVEU A INTERAÇÃO } \\
\text { DIALÓGICA. }\end{array}$ \\
\hline $\begin{array}{l}\text { A SUA PERSPECTIVA EM RELAÇÃO AO CURSO } \\
\text { E/OU A INSTITUIÇÃO MUDOU? }\end{array}$ & $\begin{array}{l}\text { AVALIAR SE A VT IMPACTOU NA RELAÇÃO DA } \\
\text { INSTITUIÇÃO COM OS ACADÊMICOS. }\end{array}$ \\
\hline
\end{tabular}

QUADRO 2 - QUESTÕES DOS OBJETIVOS PEDAGÓCICOS DO SICCC. FONTE: O AUTOR (2018 
Conforme apresentado no Quadro 2, para cada objetivo da ação de extensão há duas colunas, uma apresenta a questão de avaliação, e a outra descreve o objetivo da questão formulada.

\section{VISITA TÉCNICA}

A VT foi organizada conforme os objetivos de um dos programas de extensão do IFPR de Paranavaí, que seria promover apoio na formação técnica dos acadêmicos do CSTADS, de forma interdisciplinar, por meio de ações que permitam a aprendizagem mais próxima a realidade profissional.

Neste contexto, definiu-se que a VT deveria ser realizada em empresas produtoras de software, setores que atuam por meio de técnicas de engenharia e desenvolvimento de software, abrangendo assim, todos os objetivos do curso. Assim, iniciou-se a busca de empresas deste setor por meio das as informações disponíveis em prefeituras, associações comerciais e associações de empresas de TI da região.

Constatou-se que, Maringá é polo de desenvolvimento de software da região, sendo a $2^{\circ}$ cidade no Brasil com maior número de empresas com certificações de qualidade em tecnologia, atendendo as maiores companhias de telecomunicações do Brasil e atua ndo nos mais variados segmentos de mercado no Brasil e no exterior (CONSELHO DE DESENVOLVIMENTO ECONÔMICO, 2018). De acordo com a Great Place to Work (2018), dentre as 100 melhores empresas no Brasil para trabalhar em TI, 5 estão em Maringá.

Com o local e o ramo da empresa definidos, verificou-se quais empresas de TI da cidade de Maringá permitiriam a VT. Considerando os riscos da viagem e de atrasos entre as VT, definiu-se a realização de uma VT pela manhã e outra à tarde com duração de 2 horas cada.

Dentre as empresas disponíveis, considerou-se as que possuem experiência no setor de TI - 15 anos ou mais de atuação no setor - e adotem metodologias e processos de desenvolvimento de software atuais.

A primeira empresa escolhida foi uma fábrica de software com mais de 15 anos de experiência, eleita entre as melhores empresas de TI e Telecom para se trabalhar no Brasil e no Paraná, por 6 anos consecutivos (GREAT PLACE TO WORK, 2018). A segunda empresa atua a mais de 20 anos em desenvolvimento e metodologia ágil nos mais variados segmentos de mercado do Brasil e no exterior, com mais de 200 colaboradores. 
Após a definição das empresas, realizou-se o agendamento da visita e em conjunto com as empresas iniciou-se a definição do roteiro da VT. Considerando os objetivos da VT e o público alvo, definiu-se que a VT seria realizada para complementação didático pedagógica de disciplinas específicas do curso, com a finalidade de proporcionar aos estudantes uma visão técnica da profissão almejada, conhecendo o processo, a estrutura social, a dinâmica do espaço de trabalho de modo a contribuir no processo formativo do acadêmico.

\section{RESULTADOS}

Com base na resolução no 11, de 27 de março de 2018, 4 objetivos fundamentais a respeito das atividades de extensão do IFPR foram identificados: (1) a Indissociabilidade do Ensino, Pesquisa e Extensão; (2) Interdisciplinaridade e Interprofissionalidade; (3) Impacto na Formação do Estudante; e (4) a Interação Dialógica. Em relação ao $1^{\circ}$ objetivo, a Indissociabilidade do Ensino, Pesquisa e Extensão, 4 questões foram formuladas, nos quais, os resultados estão apresentados nos Gráficos 1 à 4 .

GRÁFICO 1-IMPORTÂNCIA DA APRENDIZAGEM. FONTE: AUTOR

GRÁFICO 2-IMPORTÂNCIA DO CURSO.FONTE: AUTOR.
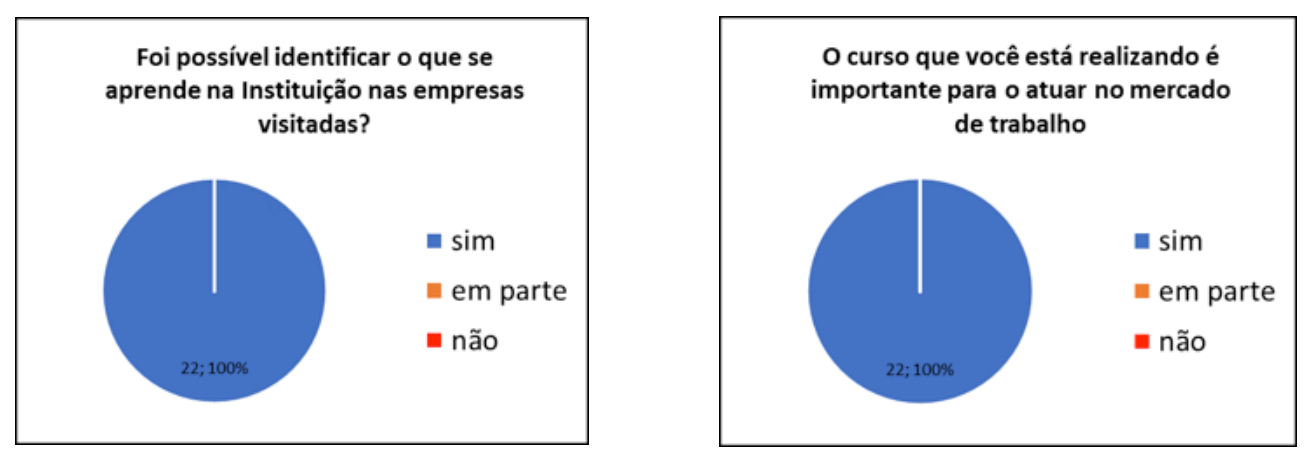

Conforme ilustra o Gráfico 1 e 2, todos os acadêmicos que participaram da VT identificaram a aplicação do que aprendem na instituição nas empresas visitadas, bem como a importância do curso que está realizado para atuar no mercado de trabalho. 
Gráfico 3 - Ambiente de Trabalho. Fonte: autor

Gráfico 4-Coerência de Ensino. Fonte: AUtor.
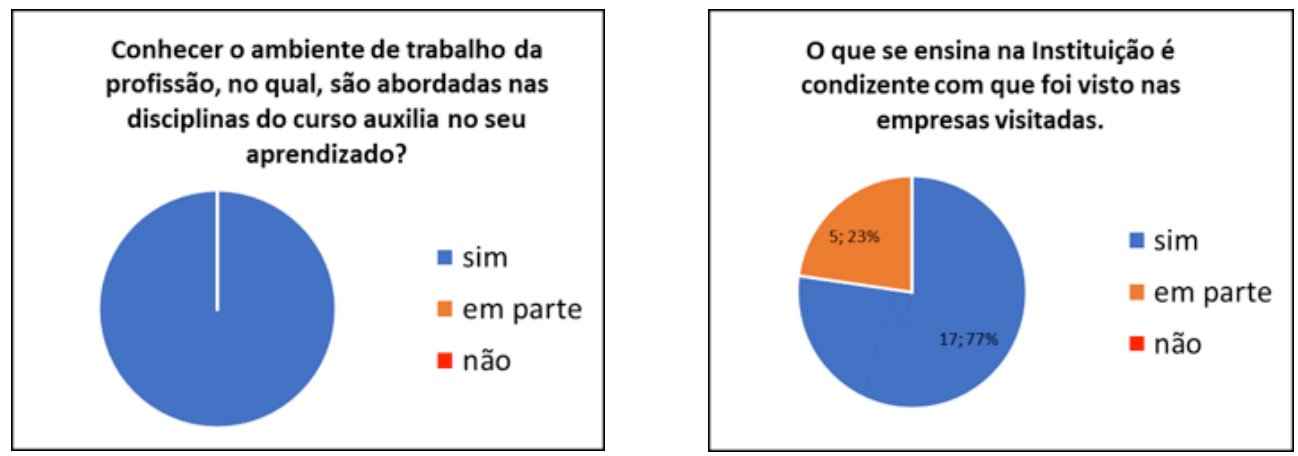

O Gráfico 3 apresenta o resultado, no qual, todos os acadêmicos afirmaram que conhecer o ambiente de trabalho da profissão, em que são abordadas as disciplinas do curso, é de suma importância. Dentre estes, 12 fundamentaram as suas afirmações, expondo que a VT auxiliou na compreensão de como as técnicas e as ferramentas estão sendo utilizadas. Os demais acadêmicos, justificaram suas respostas, explicando que foi possível associar a aprendizagem acadêmica com a realidade profissional.

Já em relação se o que está sendo ensinado na instituição é condizente ao que foi visto na VT - Gráfico 4 -, 17 acadêmicos disseram que o ensino acadêmico está condizente. Os outros 5 afirmaram que não foi possível identificar todas as tecnologias durante a VT.

A respeito do $2^{\circ}$ objetivo identificado no regimento das atividades de extensão do IFPR, a Interdisciplinaridade e Interprofissionalidade, três questões foram formuladas, nas quais, os resultados estão apresentados nos Gráficos 5 à 7 .

GRÁFICO 5-DisciplinAS dO CURSO. FONTE: AUTOR

GrÁFICO 6-INTEGRAÇÃO DO CURSO. FONTE: AUTOR.
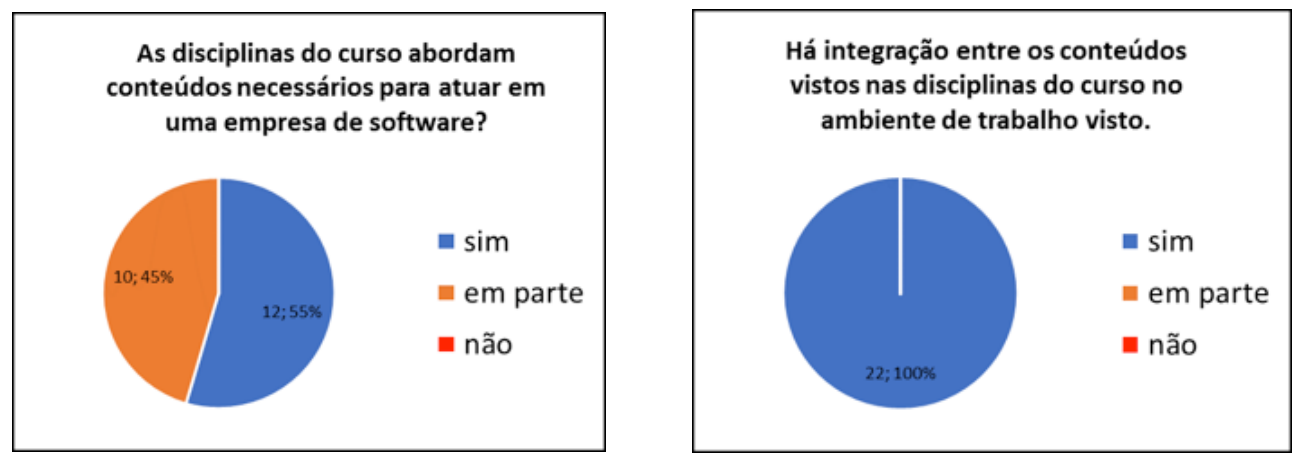
O Gráfico 5 apresenta o resultado onde foi verificado se as disciplinas do curso abordam conteúdos necessários para atuar em uma empresa de software. Doze acadêmicos afirmaram que sim, e os demais reconhecem a importância de todas as disciplinas, porém, identificaram somente as disciplinas que abordam os conteúdos técnicos.

Conforme apresentado no Gráfico 6, todos os acadêmicos perceberam a integração entre os conteúdos vistos nas disciplinas do curso no ambiente das empresas visitadas.

GrÁFICO 5-DisCIPLINAS DO CURSO. FONTE: AUTOR

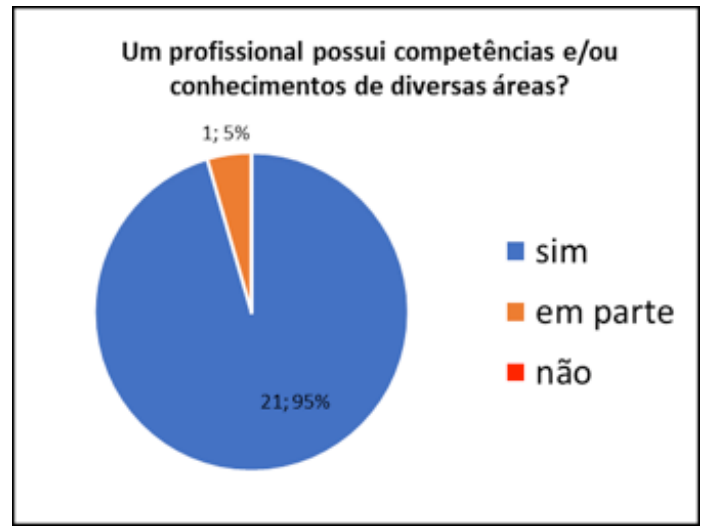

De acordo com o Gráfico 7, durante a VT, 21 dentre 22 acadêmicos identificaram que um profissional possui competências e/ou conhecimentos de diversas áreas.

Os Gráficos 8 à 12, apresentam os resultados das questões para avaliar o $3^{\circ}$ objetivo identificado no regimento das atividades de extensão do IFPR, o Impacto na Formação do Estudante.

Gráfico 8-Perspectiva dA PROFISSÃo. Fonte: AUtor

GrÁFICO 9-FORMAÇÃO.FONTE: AUTOR.
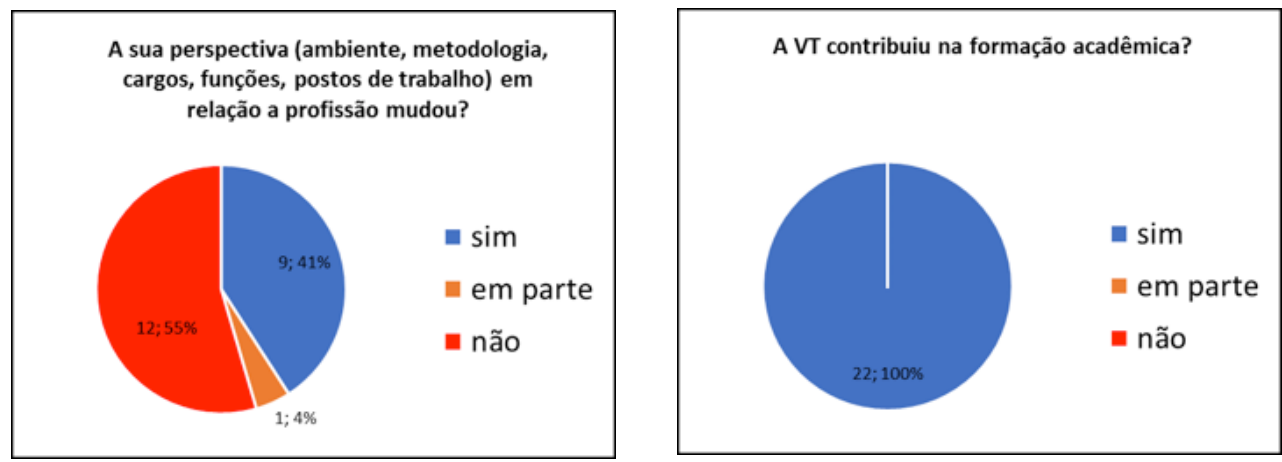
Avaliando se a perspectiva em relação a profissão mudou com a VT - Gráfico 8 -, 9 afirmaram que sim, citando o ambiente de trabalho e o tratamento dos funcionários; 1 acadêmico disse que mudou em parte, pois já atua na área utilizando ferramentas e metodologias semelhantes; e 12 acadêmicos disseram que não, pois acreditavam que o ambiente, técnicas e as ferramentas seria conforme as empresas visitadas.

O Gráfico 9 ilustra o resultado, no qual, foi avaliada se a VT contribuiu na formação acadêmica. Todos os acadêmicos afirmaram que sim, evidenciando a importância de conhecer a profissão almejada e confrontar as competências adquiridas na instituição com o que é necessário para atuar no setor.

GráFICO 10 -ESCOLHA PROFISSÃO. FONTE: AUTOR

GrÁFICO 11 - MOTIVAÇÃO ESTUdO.FONTE: AUTOR.
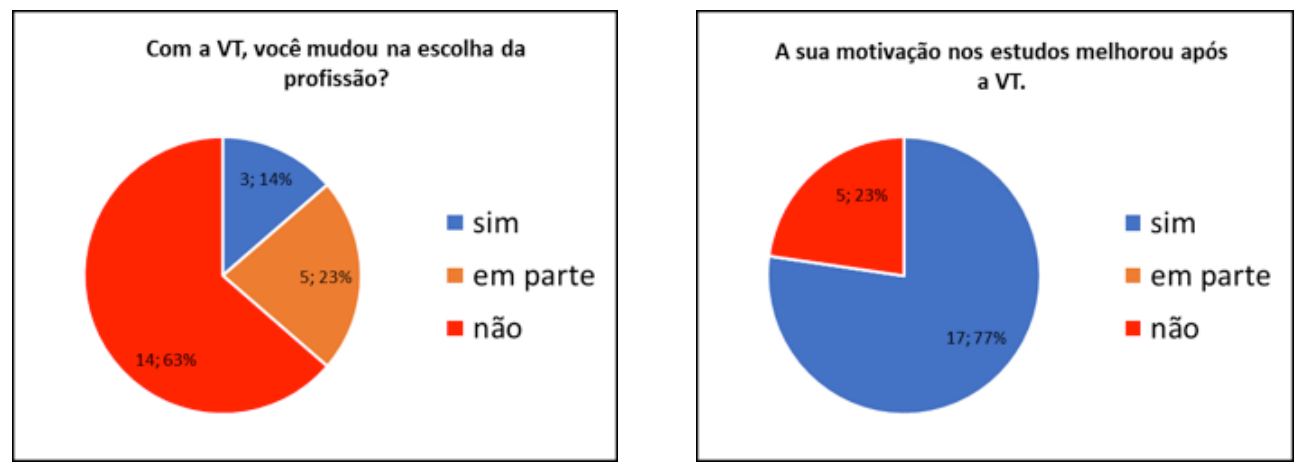

O Gráfico 10 ilustra o resultado, no qual, foi avaliado se a VT ocasionou na mudança da escolha da profissão. Três acadêmicos afirmam que sim, ressaltando que foi possível entender diversos cargos e funções. Cinco acadêmicos disseram que mudou em parte, pois identificaram uma subárea técnica do que pretendia. Quatorze acadêmicos afirmaram não ter mudado, dentre os quais, 11 expuseram que a VT reafirmou a profissão técnica almejada.

Avaliando se a motivação nos estudos melhorou após a VT - Gráfico 11 -, 17 acadêmicos afirmaram que sim, dentre estes, 15 disseram que a motivação foi causada pela apresentação de diversas oportunidades no setor de TI e a carência de mão de obra qualificada no setor. Cinco acadêmicos disseram que a VT não os motivou, dentre os quais, 4 justificaram que já eram motivados antes da VT. 
GRÁFICO 12 -FORMAÇÃO ACADÊMICA. FONTE: AUTOR

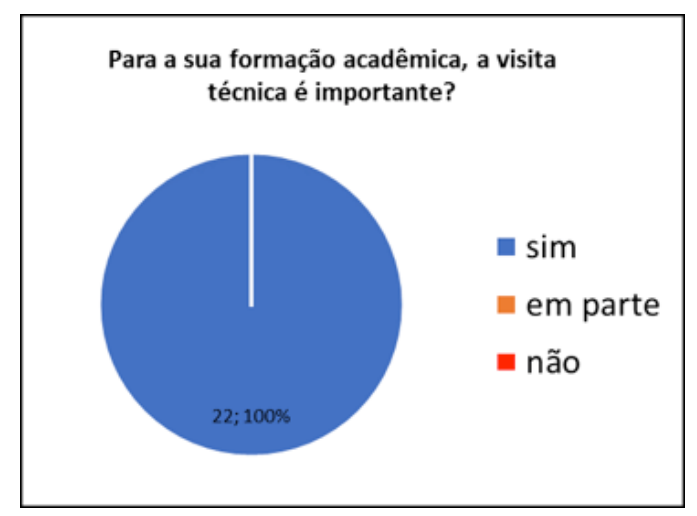

Por meio do Gráfico 13 e o Gráfico 14, verificou-se as questões para avaliar se a VT promoveu a Interação Dialógica na percepção do acadêmicos, $4^{\circ}$ objetivo identificado no regimento das atividades de extensão do IFPR.

GrÁFICO 13-EMPRESAS. Fonte: AUTOR

GRÁFICO 14 - PeRSPECTIVA INSTITUIÇÃO. FonTE: AUTOR
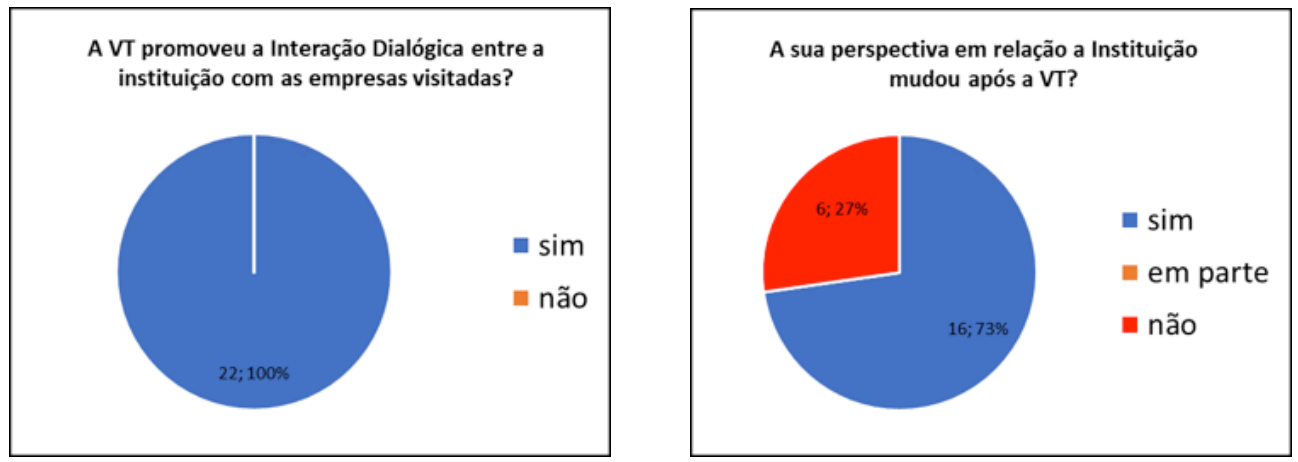

Conforme indica o Gráfico 13, todos os acadêmicos afirmaram que a VT promoveu a Interação Dialógica entre a instituição com as empresas visitadas, salientando a importância das empresas conhecerem o IFPR de Paranavaí, criando uma oportunidade de conhecer o trabalho da instituição e da formação técnica dos alunos.

O Gráfico 14 apresenta o resultado, do qual, foi verificado se a perspectiva em relação a instituição mudou após a VT. Dezesseis acadêmicos disseram que sim, sentindo-se apoiados e incentivados. Seis acadêmicos disseram que não, dentre os quais, 4 informaram que já se sentiam apoiados e motivados por parte da instituição. 


\section{CONCLUSÃO}

A atividade de extensão deve ser indissociável ao ensino, com a prática interdisciplinar e interprofissional, de forma que impacte na formação do estudante e promova a interação dialógica (CONSELHO SUPERIOR DO IFPR, 2018).

De acordo com os resultados, a VT permitiu que os acadêmicos visualizassem as aprendizagens adquiridas na instituição na realidade profissional, valorizando o ensino acadêmico, e assim, contribuindo na motivação aos estudos. Assim, conclui-se que, a VT é uma prática indissociável ao ensino, pois, os conhecimentos adquiridos na instituição de ensino relacionaram-se com o contexto profissional, apresentada de maneira relevante, substantiva e não arbitrária.

Todos os acadêmicos identificaram a integração entre os conteúdos vistos nas disciplinas do curso no ambiente das empresas visitadas, e que um profissional necessita de conhecimento de diversas profissões. Assim, constata-se que a VT buscou consistência teórica confrontando a realidade profissional, valorizando o conhecimento Interdisciplinar e Interprofissional.

Em relação ao Impacto na Formação do Estudante, os resultados indicam que na visão dos acadêmicos, a VT contribuiu na formação profissional, dentre os quais, 19 dos 22 pretendem atuar em profissões correlatas aos objetivos do curso. Desta forma, verifica-se que, a VT impactou na formação do acadêmico, ampliando o universo de referência da profissão, por meio do contato direto com as empresas de atuação, possibilitando o enriquecimento da experiência do acadêmico em termos teóricos e metodológicos.

As empresas visitadas não sabiam da existência do IFPR de Paranavaí, bem como o CSTADS. As gestoras do setor de recursos humanos das empresas, apresentaram diversas oportunidades de trabalho existentes na área de TI, expondo a falta de mão de obra qualificada e que é de suma importância conhecer as instituições que auxiliam na formação das pessoas deste setor.

Diante do exposto, constata-se que a relação da instituição com as empresas é relevante, visto que, a instituição tem o objetivo de formar profissionais qualificados e as empresas necessitam desta mão de obra qualificada. Assim, acredita-se que a VT promoveu a interação dialógica entre a instituição com a empresas, de forma que possibilite a atuação em conjunto para atender as 
demandas da sociedade. Além disso, a VT contribuiu na relação entre a instituição com os acadêmicos, que se sentiram apoiados e incentivados.

De acordo com a análise dos resultados, na perspectiva dos acadêmicos, conclui-se que a VT atendeu todos os objetivos expostos no regimento das atividades de extensão do IFPR, permitindo a articulação com o ensino na visão interdisciplinar, viabilizando a aprendizagem mais significativa. A VT compartilhou experiências e conhecimentos, ao invés de transmiti-los, uma formação que valorizou o conceito ensinado na instituição de ensino confrontada com a prática da realidade da profissão.

\section{REFERÊNCIAS}

BADARÓ, C. S. M.; FABRI, A. C. O. C.; DEUS, R. L.; DUTRA, H. S. Technical visit carried out during the formation of nursing students: a descriptive study. Online Brazilian Journal of Nursing, 2016.

BRASIL. PRESIDÊNCIA DA REPÚBLICA; CASA CIVIL, SUBCHEFIA PARA ASSUNTOS JURÍDICOS. Lei No 11.892, de 29 de dezembro de 2008. Institui a Rede Federal de Educação Profissional, Científica e Tecnológica, cria os Institutos Federais de Educação, Ciência e Tecnologia, e dá outras providências. Brasília, 2008.

CONSELHO DE DESENVOLVIMENTO ECONÔMICO. Tecnologia de Informação e Comunicação. Disponível em: http://codem.org.br. Acesso em: 22 fev. 2019.

COMISSÃO DE ELABORAÇÃO DO PPC. Projeto Pedagógico do Curso Superior de Tecnologia em Análise e Desenvolvimento de Sistemas. Instituto Federal do Paraná - Campus Paranavaí, 2013. Disponível em: http://paranavai.ifpr.edu.br/wp-content/uploads/2017/05/PPC-TADS-turmas-2016-cadastrado-no-MEC.pdf. Acesso em: 15 fev. 2019.

CONSELHO SUPERIOR DO IFPR. Aprova o regulamento das atividades de extensão do IFPR . Resolução no 11, de 27 de março de 2018. Regulamento das atividades de extensão do IFPR. Curitiba, 2018.

DUTRA, H. S.; BADARÓ, C. S. M.; COELHO, A. C. O.; BAHIA, M. T. R.; E GAMA, B. M. B. M. Utilização da visita técnica no ensino de administração em enfermagem. Revista de Enfermagem do Centro-Oeste Mineiro, 2019. 
FERKO, G. P. S.; CAMPÊLO, S. A. A visita técnica sob o olhar dos discentes do MBA em gestão de cooperativa da UFPR. Revista de Administração de Roraima, 2016.

GOMES A.; HENRIQUES J.; MENDES A. Uma proposta para ajudar alunos com dificuldades na aprendizagem inicial de programação de computadores. Revista Educação, Formação \& Tecnologias, 2008.

GREAT PLACE TO WORK. As 150 Melhores Empresas para Trabalhar. Disponível em: https://gptw.com.br/conteudo. Acesso em: 25 fev. 2019.

INSTITUTO FEDERAL DO PARANÁ (IFPR). Instrução Interna de Procedimentos do Campus IIPC n01/2014. Paranavaí: IFPR, 2014.

LIMA, L. F.; AZEVEDO, M. A. R.; AMORIN, M. V. S. Extensão Universitária na UEG: Interação Dialógica na Formação de Professores. Revista UFG, 2015.

SOUZA, D. M.; BATISTA, M. H. S.; BARBOSA, E. F. Problemas e Dificuldades no Ensino e na Aprendizagem de Programação: Um Mapeamento Sistemático. Revista Brasileira de Informática na Educação, 2016. 